\author{
荧光纳米钻石与转铁蛋白的相互作用及其在细胞内的成像应用 \\ 王东新 $a, b \quad$ 李英奇 $*, a, c$ 杨斌盛 $*, a$ \\ ( ${ }^{a}$ 山西大学分子科学研究所 化学生物学与分子工程教育部重点实验室 太原 030006) \\ $\left({ }^{b}\right.$ 山西医科大学公共卫生学院 太原 030001) \\ ( ${ }^{c}$ 山西大学化学化工学院 太原 030006)
}

\begin{abstract}
摘要 通过电导返滴定法测定经强酸氧化后的苂光纳米钻石 $(\mathrm{FND}$, 约 $140 \mathrm{~nm}$ )表面羧基含量为 $126 \mu \mathrm{mol} / \mathrm{g}$, 占表面原 子数的 $29.7 \%$. 对 FND 物理吸附人转铁蛋白(hTf)进行了研究, 其吸附行为符合 Langmuir 等温吸附, 在 PBS(pH 7.4)中 最大吸附量为 (176.46 \pm 2.13$) \mu \mathrm{g} / \mathrm{mg}$, 同时研究了 $\mathrm{pH}$ 对 FND 吸附 $\mathrm{hTf}$ 的影响, 发现在 $\mathrm{pH}$ 等于 $\mathrm{hTf}$ 的等电点附近有最大 吸附. 利用激光共聚焦和流式细胞仪对 FND 和 FND-hTf 内吞到人肝癌细胞(HepG2)的差异进行了定性和定量分析, 结 果得到 FND-hTf 比 FND 容易内吞到细胞中, 利于胞内成像.

关键词 荧光纳米钻石; 转铁蛋白; Langmuir 等温吸附; 细胞成像
\end{abstract}

\title{
Interaction between Fluorescent Nanodiamond and Human Transferrin and Intracellular Imaging
}

\author{
Wang, Dongxin ${ }^{a, b} \quad$ Li, Yingqi*, ${ }^{*, c} \quad$ Yang, Binsheng*, \\ ( ${ }^{a}$ Key Laboratory of Chemical Biology and Molecular Engineering of Ministry of Education, Institute of Molecular Science, \\ Shanxi University, Taiyuan 030006, China) \\ $\left({ }^{b}\right.$ Public Health College, Shanxi Medical University, Taiyuan 030001, China) \\ ( ${ }^{c}$ College of Chemistry and Chemical Engineering, Shanxi University, Taiyuan 030006, China)
}

\begin{abstract}
Nanodiamond (ND), as a member of carbon nanomaterials family, has recently received increasing attention for their potential applications as imaging and drug delivery agents. Due to several charming properties, such as surface functionalization capability, biocompatibility and chemical stability, ND demonstrates high affinity to biomolecules. In this work, the adsorptive behavior of oxidized fluorescent nanodiamond (FND, with a size of $c a .140 \mathrm{~nm}$ ) for hTf was investigated. The amount of surface carboxylic acid on oxidized FND was $126 \mu \mathrm{mol} / \mathrm{g}$ determined by conductometric backward titration. This amount is $29.7 \%$ of the total surface atoms. HTf physically adsorbed on the surface of FND (FND-hTf) in PBS (pH7.4) shows that the isothermyal adsorption behavior is coincident with Langmuir model and the maximum adsorbed amount is $(176.46 \pm 2.13) \mu \mathrm{g} / \mathrm{mg}$. ND coated human transferrin (hTf) can improve the dispersity and stability compared to pristine FND under a physiological environment or in cell culture medium observed through optical microscope and be more suitable for biomedicine applications. Simultaneously, $\mathrm{pH}$ effect on hTf adsorbed onto FND is also inquiried. The result exhibits that FND has the greatest adsorption capacity for hTf close to the isoelectric point. Owing to the negatively charged nitrogenvacancy $(\mathrm{N}-\mathrm{V})^{-}$defect centers, FND can absorb strongly at $c a .560 \mathrm{~nm}$ and emit fluorescence efficiently at $c a .700 \mathrm{~nm}$, which can be well quantitatively and qualitatively analyzed by flow cytometry and confocal fluorescence images. In vitro experiments of human liver cancer cells (HepG2) uptake of nanoparticles display that FND-hTf nanoparticles are more easily endocytosed than that of pristine FND. So FND-hTf is conducive to cell imaging. Furthermore, flow cytometry assay indicates cellular uptake of FND-hTf reached a plateau at about $8 \mathrm{~h}$ and the uptake half-life is about $(1.41 \pm 0.22) \mathrm{h}$ at a particle concentration of $100 \mu \mathrm{g} / \mathrm{mL}$. The results obtained by confocal fluorescent images display that the FND-hTf nanoparticles locate in the cytoplasm and mainly distribute on the surface of the nucleus, however, can not enter the nucleus.

Keywords fluorescent nanodiamond; human transferrin; Langmuir isothermy adsorption; cell imaging
\end{abstract}

\section{1 引言}

近年来, 纳米钻石 (nanodiamond, ND) 在生物医药中
的应用引起广泛关注 ${ }^{[1,2]}$. 研究结果表明, 爆炸合成的 $\mathrm{ND}$ 生物相容性好, 对多种细胞无明显细胞毒性 ${ }^{[3 \sim 8]}$; 经 强酸氧化处理后表面富含含氧基团，易于修饰. Chang

*E-mail: wkyqli@sxu.edu.cn; yangbs@sxu.edu.cn

Received January 31, 2013; published March 15, 2013.

Supporting information for this article is available free of charge via the Internet at http://sioc-journal.cn.

Project supported by the National Natural Science Foundation of China (Grant No. 21071091), the Shanxi Provincial Natural Science Foundation (Grant No. 2009011012-3), Shanxi Scholarship Council of China (201011) and the Youth Foundation of Shanxi Medical University (02201114).

项目受国家自然科学基金(No. 21071091)、山西省自然科学基金(No. 2009011012-3)、山西省归国留学基金(No. 201011)和山西医科大学青年基金(No. 02201114)资助. 
等 ${ }^{[9]}$ 通过离子辐射含氮量较高的 Type Ib 型 ND, 再经过 退火处理后得到可发约 $700 \mathrm{~nm}$ 的红色苂光纳米钻石 (Fluorescent nanodiamond, FND), 可避免生物体的自发 荧光的干扰, 与有机苂光染料相比没有光漂白现象, 表 明其具有优良的光学稳定性. 基于以上优点, FND 可被 作为药物载体和生物标记. 但是由于 FND 本身在生理 条件和细胞培养介质中容易聚集, 影响其在生物领域中 的应用 ${ }^{[10]}$, 特别是作为生物标记时影响其细胞的内吞, 无法均匀标记到每个细胞中. 因此, 利用共价键和非共 价键的修饰方法来提高 ND 的分散性做了大量的研究, 多使用一些亲水性的化合物如聚乙二醇或蛋白等 ${ }^{[11 ~ 17]}$.

人体内存在的人血清转铁蛋白(human transferrin, hTf)主要通过与细胞膜表面的转铁蛋白受体作用介导 $\mathrm{Fe}^{3+}$ 运输和调控 ${ }^{[18]}$, 研究表明, 多数肿瘤细胞表面 hTf 受体的表达量高于正常细胞, 可作为靶向蛋白用于肿瘤 的治疗 ${ }^{[19]}$. 通过生物偶联的方法将 FND 与 hTf 共价偶 联, 由 hTf 受体介导的网格蛋白途径进入 Hela 细胞(宫 颈癌细胞), 在胞内成像 ${ }^{[20 ~ 22]}$. 但是生物偶联可能导致 hTf 变性以及因为有部分物理吸附的 hTf 存在, 共价偶 联量无法准确确定. 本文对 FND与 hTf 的物理吸附作用 进行了定量的研究, 同时以人的肝癌细胞(HepG2)为模 型, 对 FND 和 FND-hTf 的细胞内吞成像及内吞量进行 了研究.

\section{2 结果与讨论}

\subsection{FND 表面羧酸含量的测定}

FND 经强酸氧化处理 ${ }^{[23]}$, 表面富含含氧基团, 如羧 基、羰基、酮基、羟基、内酯等. 这些基团的存在，易 于将不同的活性分子修饰到 FND 表面, 尤其是表面羧 基基团的存在，易于将蛋白、酶等修饰到其表面. 为了 定量确定钻石表面羧基的含量, 我们采用电导返滴定法 测定 FND 表面的羧基含量. 从图 1 曲线可以看到, 随着 $\mathrm{HCl}$ 含量的增加, 电导率先降低, 随后保持恒定值, 再 上升. 第一区域直线表示 $\mathrm{NaOH}$ 溶液的电导率, 随着盐 酸的加入, 电导率降低. 第三区域直线表示过量 $\mathrm{HCl}$ 的 电导率, 随着盐酸的加入, 其电导率上升. 第二区域是 FND 表面羧基的电导部分, 由于 FND 表面氧化的羧酸 为弱酸性, 与 $\mathrm{NaOH}$ 反应生成弱酸强碱盐, 当用盐酸滴 定时 FND 表面的 $\mathrm{Na}^{+}$被 $\mathrm{H}^{+}$取代, 并且 FND 粒径较大, 迁移较慢, 其电导率变化较平缓. 根据第二区域盐酸的 用量为 $3.15 \mu \mathrm{mol}$, 可得到 FND 表面的羧酸含量为 126 $\mu \mathrm{mol} / \mathrm{g}$. 根据许向阳 ${ }^{[24]}$ 提出的纳米钻石的理论模型计 算, 得到 $140 \mathrm{~nm}$ 的 FND 体积为 $1.43 \times 10^{6} \mathrm{~nm}^{3}$, 表面原 子数为 $1.13 \times 10^{6}$. 根据 FND 的密度 $3.1 \mathrm{~g} / \mathrm{cm}^{3}$, 得到每 个 FND 粒子上含有 $3.36 \times 10^{5}$ 个羧基, 占表面原子数的 $29.7 \%$. 如此高的表面羧酸量有利于静电吸附带正电的 分子或蛋白.

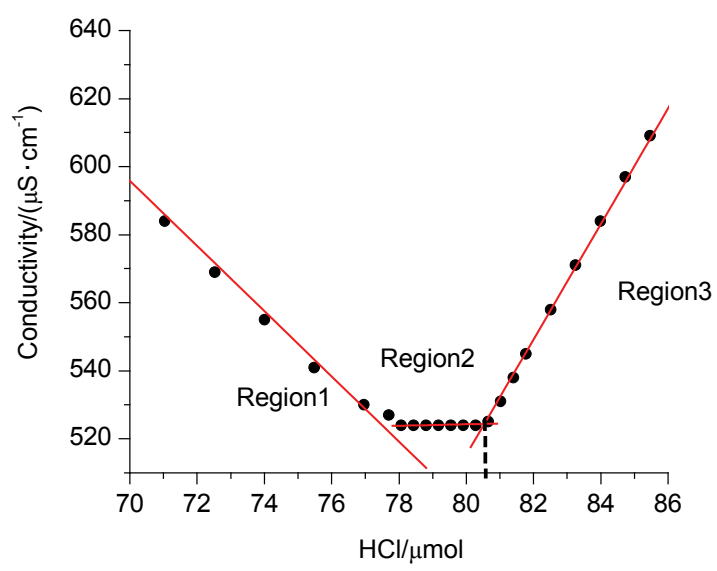

图 1 电导滴定法测定 FND 表面羧酸含量

Figure 1 Conductometric titration of amount of surface carboxylic acid on oxidative-acid-treated FND

\subsection{FND 对 hTf 吸附的测定}

根据 Bradford 法测定加入的 hTf 标准溶液在 $595 \mathrm{~nm}$ 处的吸光度 $\mathrm{A}$ 并绘制标准曲线(图 S1, 支持信息), 线性 回归得到方程为 $y=0.02206 x-0.0007$, 相关系数 $r=$ 0.992 , 相关性较好, 可用此法对 hTf 进行定量测定.

FND 对 hTf 吸附曲线如图 2 所示. 在一定范围内, FND 对 hTf 的吸附量随 hTf 量的增加而增加, 当 hTf 超 过一定量, 随着 hTf 量的增加 FND 对 hTf 的吸附量不再 增加而趋于恒值, 即此时 FND 吸附 hTf 已达到饱和. FND 吸附 hTf 呈现两种状态, 吸附饱和前的近乎线性吸 附和饱和吸附, 符合 Langmuir 等温吸附曲线, 由图可 见, 当加入的 $\mathrm{hTf}$ 量为 $750 \mu \mathrm{g}$ 时, 吸附量达到最大为 $(176.46 \pm 2.13) \mu \mathrm{g} / \mathrm{mg}$. 此后随 hTf 量的增加 FND 对 hTf 的吸附量不再增加. 根据 Langmuir 等温吸附公式 ${ }^{[11]}$

$$
\Gamma=\frac{K_{\mathrm{a}} C}{1+K_{\mathrm{a}} C}
$$

可计算吸附常数, 式中 $\Gamma$ 为占据位点和饱和位点的比 值, $K_{\mathrm{a}}$ 为吸附常数, $C$ 为 $\mathrm{hTf}$ 的平衡浓度. 计算得到吸附

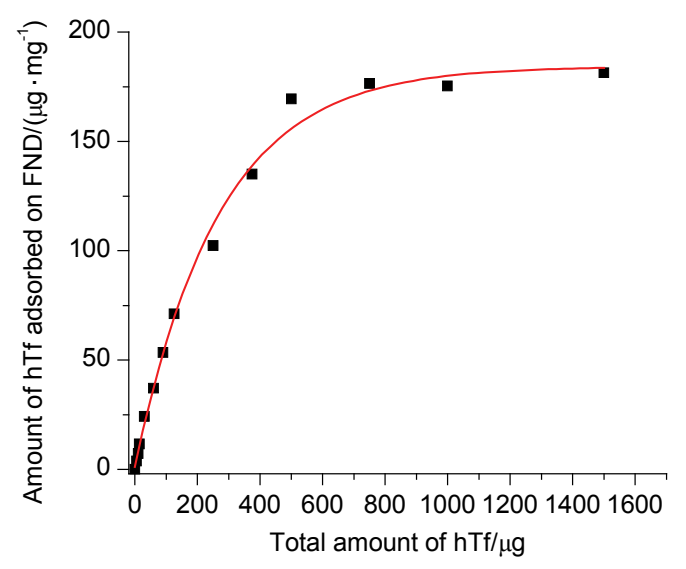

图 2 FND 在 PBS (pH 7.4)中对 hTf 的吸附

Figure 2 Adsorption curve of hTf on FND in PBS (pH 7.4) 
常数 $K_{\mathrm{a}}$ 为 $(1.78 \pm 0.29) \times 10^{6} \mathrm{~L} \cdot \mathrm{mol}^{-1}$. FND 与 hTf 的物理 吸附作用主要有静电作用、氢键、范德华力等 ${ }^{[23]}$.

此外, 研究了 $\mathrm{pH}$ 对 FND 吸附 hTf 的影响, 如图 3 所示, 在 $\mathrm{pH}$ 为 5.5 时 FND 对 hTf 的吸附量最大. hTf 的 等电点为 $5.6 \sim 5.8, \mathrm{pH}$ 在等电点附近 FND 对 $\mathrm{hTf}$ 的吸附 能力最大, 这与 Chang 等 ${ }^{[25]}$ 研究纳米钻石物理吸附牛血 清白蛋白和 $\alpha$-乳白蛋白的结论一致. 这可能是因为 $\mathrm{hTf}$ 在等电点时溶解度最小, 从而使其与 FND 的疏水作用 力增大, 吸附能力增强.

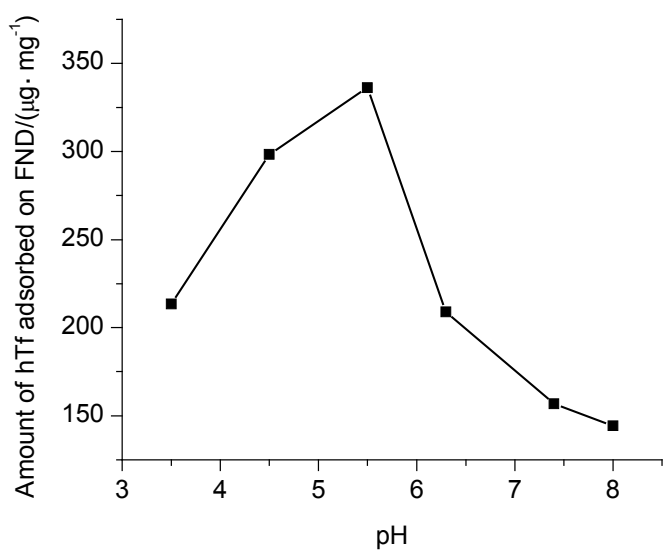

图 $3 \mathrm{FND}$ 在不同 $\mathrm{pH}$ 条件下对 $\mathrm{hTf}$ 的吸附

Figure 3 Amount of hTf adsorbed on FND as a function of $\mathrm{pH}$

为确定转铁蛋白吸附在纳米钻石上, 用激光粒度分 析仪分别测定了 FND 和 FND-hTf 的粒径和 Zeta 电位, 结果见表 $\mathrm{S} 1$ (支持信息). 未修饰的 FND 平均粒径为 $(148.96 \pm 5.46) \mathrm{nm}$, 吸附 $\mathrm{hTf}$ 后平均粒径成为

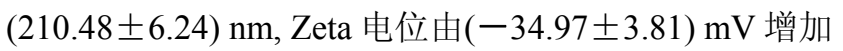
到( - 17.72 \pm 2.64$) \mathrm{mV}$, 表明 hTf 的确吸附到 FND 表面 上. 通过光学显微镜观察了 FND 和 FND-hTf 在含 10\% 的胎牛血清(Fetal Bovine Serum, FBS)的细胞培养基 Dulbecco's Modified Eagle Medium, DMEM)中的分散, 结果表明(图 S2, 支持信息), FND-hTf 在细胞培养液中 的分散性明显优于 FND.

\section{3 细胞对 FND 和 FND-HTf 的摄入研究}

FND 通过约 $560 \mathrm{~nm}$ 的激光激发可发射约 $700 \mathrm{~nm}$ 的 荧光(图 S3, 支持信息), 发光中心主要为带负电的氮-空 位缺陷中心 $(\mathrm{N}-\mathrm{V})^{-}$引起的 ${ }^{[9]}$, 已有文献测定其荧光量子 产率为 $\approx 1$, 室温下荧光寿命为 $11.6 \mathrm{~ns}^{[26]}$. 研究表明在 FND 表面修饰后不影响它的荧光性质和荧光寿命 ${ }^{[20]}$.

鉴于以上优点, FND 可作为荧光探针跟踪药物分子 或蛋白与细胞的相互作用研究. 但是 FND 本身在生理 条件或细胞培养液中容易聚集, 使其进入细胞的量有 限，从而限制其生物应用. 通过物理吸附 hTf 后可降低 FND 的表面电位, 提高其分散性, 有利于细胞的摄取. 用流式细胞仪测定了 HepG2 细胞内吞纳米颗粒后胞内 FND 的荧光强度, 可间接反映细胞对纳米颗粒的内吞
量. 如图 4 所示, 对细胞内吞纳米颗粒的动力学研究表 明, HepG2 细胞内吞 FND-hTf 在 $8 \mathrm{~h}$ 达到最大, 以一级 动力学拟合得到其内吞达到最大量的一半时的时间为 $(1.41 \pm 0.22) \mathrm{h}$. 与此相比, 细胞内吞 FND 的量明显小于 内吞 FND-hTf 的量, 这是因为 FND 和 FND-hTf 的表面 电位不同及其与细胞作用的方式不同而造成的. 由于 FND 表面富含羧基而带负电荷, 在细胞培养基中易于 吸附阳离子, 如带正电的氨基酸和胎牛血清等, 促进其 聚集而阻止了细胞的内吞. 而 FND吸附hTf后表面电位 绝对值减小，减弱了与培养基中各成分的作用，并且利 用 $\mathrm{hTf}$ 与细胞表面转铁蛋白受体的特异性作用, 因而增 加了细胞对 FND-hTf 纳米颗粒的内吞. 为进一步确定纳 米颗粒进入细胞的位置, 使用激光共聚焦扫描显微镜观 察 HepG2 细胞对 FND(图 5A) 和 FND-hTf (图 5B) 的摄取. 如图 5 所示, 红色为 FND 的荧光, 蓝色为 H33258 染细 胞核的荧光. 由图 5 可见, FND 在含 $10 \%$ FBS 的 DMEM 中与细胞孵育 $5 \mathrm{~h}$, 细胞摄入 FND 的量较少, 而同样条 件FND-hTf进入细胞的量明显增多, 主要分布在细胞质 且位于细胞核周围，不能进入细胞核。表明 FND 吸附 hTf 后有利于 HepG2 细胞的内吞. 这与流式细胞术测定 的结果(见图 4)一致, 这个结果表明 FND-hTf 可作为潜

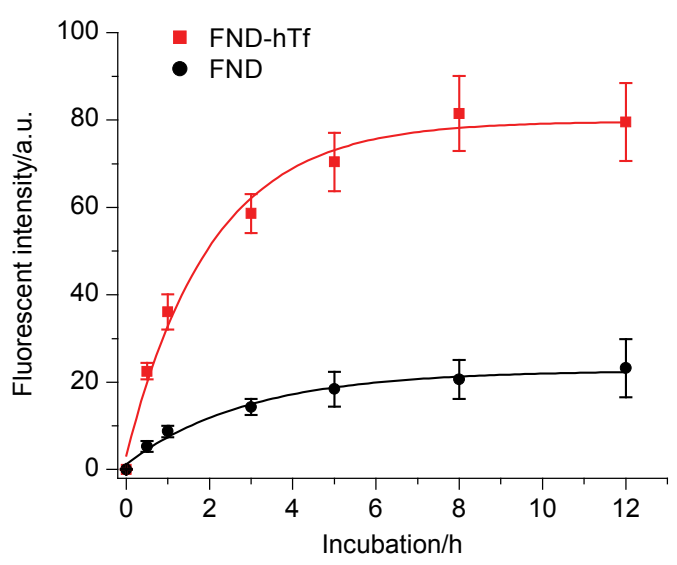

图 4 流式细胞术测定细胞摄入 FND 和 FND-hTf 动力学

Figure 4 Kinetics of the cellular uptake of FND and FND-hTf at a particle concentration of $100 \mu \mathrm{g} / \mathrm{mL}$ by flow cytometry
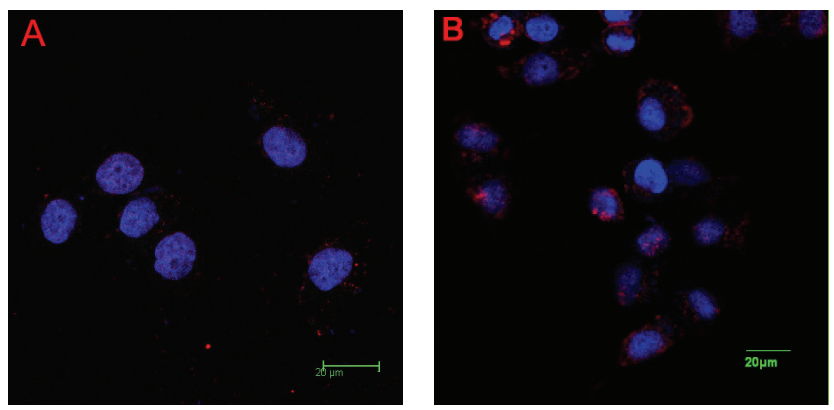

图 5 激光共聚焦显微镜测定胞内 FND-hTf 的位置

Figure 5 Intracellular location of FND-hTf after endocytosis in living HepG2 cells by the confocal fluorescence microscopy (bars, $20 \mu \mathrm{m}$ ) (A) FND as a control; (B) FND-hTf. The cell nucleus dyed with H33258, the nano FND with red fluorescence 
在的载药载体输送药物到达细胞内部, 同时进行胞内跟 踪，可以为靶向治疗肿瘤提供一定的理论依据.

\section{3 结论}

纳米钻石作为碳纳米材料的成员之一, 具有良好生 物相容性, 在生物领域的应用引起广泛关注. 利用电导 滴定法对经强酸氧化纯化后的爆炸合成的纳米钻石的 表面羧酸含量进行测定, 得到表面富含羧酸, 含量为 $126 \mu \mathrm{mol} / \mathrm{g}$, 占表面原子数的 $29.7 \%$. 如此多的羧酸含 量有利于 FND 的表面共价修饰以及对一些药物、蛋白 等生物分子的吸附. 本文对 FND 物理吸附 HTf 进行了 系统的研究, 发现其吸附行为符合 Langmuir 等温吸附, 在 PBS ( $\mathrm{pH}$ 7.4)中最大吸附量为 $(176.46 \pm 2.13) \mu \mathrm{g} / \mathrm{mg}$, 同时研究了 $\mathrm{pH}$ 对 FND 吸附 HTf 的影响, 在 $\mathrm{pH}$ 等于 $\mathrm{hTf}$ 的等电点附近有最大吸附, 可能是因为 hTf 在等电点时 溶解度最小, 从而使其与 FND 的疏水作用力增大, 吸附 能力增强. 利用激光共聚焦和流式细胞仪对 FND 和 FND-hTf 内吞到 HepG2 细胞的差异进行了定性和定量 分析, 结果得到FND-hTf 比FND容易内吞到细胞中, 利 于胞内成像.

\section{4 实验部分}

\section{1 电导滴定法测定 NDs 表面的羧基含量}

取配制好的 $0.1 \mathrm{~mol} / \mathrm{L} \mathrm{NaOH}$ 溶液 $5 \mathrm{~mL}$ 于试管中, 加 $50 \mu \mathrm{L}$ 酚酞和 $25 \mathrm{mg}$ FND, 将电导池插入此溶液中, 测出其电导率, 随后逐次加入不同量的 $0.1 \mathrm{~mol} / \mathrm{L} \mathrm{HCl}$ 溶液, 待稳定后记录读数, 以 $\mathrm{HCl}$ 溶液用量为横坐标, 电导率为纵坐标作图.

\section{2 考马斯亮蓝法(Bradford 法)测定 hTf}

考马斯亮蓝的配制: 称取 $100.2 \mathrm{mg}$ 考马斯亮蓝

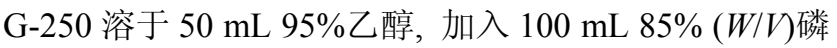
酸, 将溶液用水稀释到 $1000 \mathrm{~mL}$. 试剂的终浓度为 $0.01 \%$ 考马斯亮蓝 G-250, 4.7\% 乙醇和 $8.5 \%(\mathrm{~W} / \mathrm{V})$ 磷酸.

标准曲线绘制: 精密称取 hTf $25 \mathrm{mg}$, 加 PBS(pH7.4)溶解定容于 $10 \mathrm{~mL}$ 容量瓶中 (2.5 mg/mL), 分别取配好的 hTf $0,10,20,40,60,80,100 \mu \mathrm{L}$, 加 PBS 至 $100 \mu \mathrm{L}$, 加 $4.9 \mathrm{~mL}$ 蛋白试剂, 漩涡混匀 $1 \mathrm{~min}, 5 \mathrm{~min}$ 后于 $595 \mathrm{~nm}$ 处测定吸收值 $A$, 以 hTf 浓度 $C$ 为横坐标, $A$ 为纵坐标, 进行线性回归并绘制标准曲线.

取不同量的 hTf 与 $1 \mathrm{mg} / \mathrm{mL}$ FND 置于 PBS (pH7.4) 超声分散形成悬浊液于振荡器上混合过夜, 于 15000 $\mathrm{r} / \mathrm{min}$ 离心 $10 \mathrm{~min}$, 用 $\mathrm{PBS}$ 清洗沉淀三次, 收集每次的离 心上清液, 分别测定其吸光度值, 根据标准曲线公式求 出游离 $\mathrm{hTf}$ 浓度, 加入 $\mathrm{hTf}$ 总量与上清液中游离的 $\mathrm{hTf}$ 量之差, 即为 FND 吸附 hTf 的量, 据此绘制 hTf 吸附曲 线. 所得沉淀即为 FND-hTf.

在不同 $\mathrm{pH}$ 条件下分散 $1 \mathrm{mg} / \mathrm{mL}$ FND 悬液, 分别加
入 $1 \mathrm{mg}$ 的 hTf, 测定 FND 在不同 $\mathrm{pH}$ 条件下对 hTf 的吸 附量.

将一定质量的纳米粒子 FND 和 FND-hTf (pH7.4 制 备)置于蒸馏水并超声分散 $30 \mathrm{~min}$, 使之成为合适浓度 的悬浊液, 取适量悬浊液, 用激光粒度分析仪及高分辨 Zeta 电位, 分别测定纳米颗粒的粒径及表面 Zeta 电位.

\section{3 细胞对 FND 及 FND-hTf 的摄取研究}

取对数生长期 HepG2 细胞以 $1.5 \times 10^{5} /$ dish 的密度 接种于 $35 \mathrm{~mm}$ 培养血中, 培养 $16 \mathrm{~h}$ 后, 分别加入 100 $\mu \mathrm{g} / \mathrm{mL}$ FND 和 FND-hTf于 $37{ }^{\circ} \mathrm{C}$ 孵育不同的时间 $(0 \sim 12$ h), 用流式细胞仪测定细胞内吞纳米颗粒随时间的变 化.

将 $100 \mu \mathrm{g} / \mathrm{mL}$ FND 和 FND-hTf 分别置于含盖玻片 的培养血中, 于 $37{ }^{\circ} \mathrm{C}$ 孵育 $5 \mathrm{~h}$, 用 PBS 洗去细胞表面 FND，加盖玻片的培养盘用 $4 \%$ 多聚甲醛室温固定 8 min, 再用 H33258 染核 $15 \mathrm{~min}$, 冷 PBS 冲洗细胞三次, 取出盖玻片置于载玻片上，于激光扫描共聚焦显微镜下 观察并拍照(激发波长为 $405 \mathrm{~nm}$, 发射波长 $460 \mathrm{~nm}$ 测定 细胞核，激发波长为 $535 \mathrm{~nm}$ ，接受波长 600 700 nm 测 定 FND).

\section{致谢}

感谢台湾中央研究院 H. C. Chang 教授无偿提供的 苂光纳米钻石.

\section{References}

[1] Mochalin1, V. N.; Shenderova, O.; Ho, D.; Gogotsi1, Y. Nat. Nanotechnol. 2012, 7,11.

[2] Xing, Y.; Dai, L. Nanomedicine 2009, 4, 207.

[3] Lien, Z. Y.; Hsu, T. C.; Liu, K. K.; Liao, W. S.; Hwang, K. C.; Chao, J. I. Biomaterials 2012, 33, 6172.

[4] Chao, J.-I.; Perevedentseva, E.; Chung, P.-H.; Liu, K.-K.; Cheng, C.-Y.; Chang, C.-C.; Cheng, C.-L. Biophys. J. 2007, 93, 2199.

[5] Huang, H.; Pierstorff, E.; Osawa, E.; Ho, D. Nano Lett. 2007, 7, 3305.

[6] Schrand, A. M.; Huang, H.; Carlson, C.; Schlager, J. J.; Osawa, E.; Hussain, S. M.; Dai, L. J. Phys. Chem. B 2007, 111, 2

[7] Schrand, A. M.; Dai, L.; Schlager, J. J.; Hussain, S. M.; Osawa, E. Diam. Relat. Mater. 2007, 16, 2118.

[8] Liu, K. K.; Cheng, C. L.; Chang, C. C.; Chao, J.-I. Nanotechnology 2007, 18, 325102 .

[9] Fu, C.-C.; Lee, H.-Y.; Chen, K.; Lim, T.-S.; Wu, H.-Y.; Lin, P.-K.; Wei, P.-K.; Tsao, P.-H.; Chang, H.-C.; Fann, W. PNAS 2007, 104, 727.

[10] Kruger, A.; Kataoka, F.; Ozawa, M.; Fujino, T.; Suzuki, Y.; Aleksenskii, A. E.; Vul, A. Y.; Osawa, E. Carbon 2005, 43, 1722.

[11] Lora Huang, L.-C.; Chang, H.-C. Langmuir 2004, 20, 5879.

[12] Wang, H.-D.; Yang, Q.; Niu, C. H. Diam. Relat. Mater. 2010, 19, 441.

[13] Zhao, L.; Takimoto, T.; Ito, M.; Kitagawa, N.; Kimura, T.; Komatsu, N. Angew. Chem. Int. Ed. 2011, 50, 1388.

[14] Ushizawa, K.; Sato, Y.; Mitsumori, T.; Machinami, T.; Ueda, T.; Ando, T. Chem. Phys. Lett. 2002, 351, 105.

[15] Krueger, A.; Stegk, J.; Liang, Y.; Lu, L.; Jarre, G. Langmuir 2008 24,4200 .

[16] Man, H. B.; Lam, R.; Chen, M.; Osawa, E.; Ho, D. Phys. Status Solidi $A$ 2012, 209, 1811

[17] Fu, Y.; An, N.; Zheng, S.; Liang, A.; Li, Y. Diam. Relat. Mater. 2012, 21,73 . 
[18] Lepelletier, Y.; Camara-Clayette, V.; Jin, H.; Hermant, A.; Coulon, S.; Dussiot, M.; Arcos-Fajardo, M.; Baude, C.; Canionni, D.; Delarue, R.; Brousse, N.; Benaroch, P.; Benhamou, M.; Ribrag, V.; Monteiro, R. C.; Moura, I. C.; Hermine, O. Cancer Res. 2007, 67, 1145 .

[19] Wang, J.; Tian, S.; Robby, A. P.; Napier, M. E.; DeSimone, J. M. J. Am. Chem. Soc. 2010, 132, 11306.

[20] Weng, M.-F.; Chiang, S.-Y.; Wang, N.-S.; Niu, H. Diam. Relat. Mater. 2009, 18, 587.

[21] Li, Y. Q.; Zhou, X. P. Diam. Relat. Mater. 2010, 19, 1163.
[22] Li, Y. Q.; Zhou, X. P.; Wang, D. X.; Yang, B. S.; Yang, P. J. Mater. Chem. 2011, 21, 16406.

[23] Huang, H.; Dai, L.; Wang, D. H.; Tan, L.-S.; Osawa, E. J. Mater. Chem. 2008, 18, 1347.

[24] Xu, X. Y. Ph.D. Dissertation, Central South University, Changsha, 2007. (许向阳, 博士论文, 中南大学, 长沙, 2007.)

[25] Tzeng, Y.-K.; Faklaris, O.; Chang, B.-M.; Kuo, Y.; Hsu, J.-H.; Chang, H.-C. Angew. Chem. Int. Ed. 2011, 50, 2262.

[26] Collins, A. T.; Thomaz, M. F.; Jorge, M. I. B. J. Phys. C: Solid State Phys. 1983, 16, 2177.

(Cheng, B.; Lu, Z.) 